\title{
Highly Selective Dual-Mode Microstrip Bandpass Filters Using Triangular Patch Resonators
}

\author{
K.G. Avinash, I. Srinivasa Rao \\ School of Electronics Engineering, VIT University, Vellore, India \\ E-mail: avinashk.ng2013evit.ac.in
}

\begin{abstract}
In this paper, highly selective dual-mode microstrip bandpass filters are proposed using horizontal and vertical slots formed on the surface of a triangular patch. Slots are responsible for splitting and coupling of dual degenerate modes and also facilitate in controlling their resonant frequencies by varying the dimensions of the slots. Horizontal and vertical slots reroute the direction of the flow of current on the surface of the patch, which results in switching of transmission zeros. Two 2-pole and one 4-pole dual-mode filters are designed, simulated and fabricated. Two-pole filters have transmission zeros on side of the passband and four-pole filter have transmission zeros on both sides of the passband. The designed filters exhibit a fractional bandwidth of less than 7 $\%$ and there is a good agreement between simulated and measured results.
\end{abstract}

\section{Introduction}

Microstrip filters with asymmetric frequency response on either side of the passband are needed in modern mobile and wireless communication systems. These filters have better selectivity, sensitivity and thereby improve the efficiency of the spectrum utilization. The filters of this kind find specific applications in duplexers and multiplexers. Generally, asymmetric filters are realized by using cross-coupled filter configurations [1-3]. Single mode HTS thin film filters using pseudo-interdigital coupled lines are employed to achieve asymmetrical rejection performance [1]. Microstrip square open loop resonators [2] are used to introduce either positive or negative cross coupling. This causes finite attenuation pole to appear either in lower or upper side of the passband. In [3] closed square loop resonator with varied perturbation is used to achieve asymmetric frequency response utilizing dual degenerate modes.

Dual-mode resonators are preferred as the number of resonators required will become half for a given degree which further reduces the size of the filter. The basic geometries used for the realization of dual-mode filters are loop [4-7] and patch resonators. Patch resonators employing circular, square and triangular shapes are reported [1], [8-9]. Patch resonators have many interesting features such as high power handling capability, lower conducting losses and ease of fabrication at millimeter waves. Highly selective bandpass filters were realized by using two different resonant modes of triangular patch with cascading topology [8]. Dual-mode operation of equilateral triangular patch resonator was theoretically studied in [10], and they proved that dual degenerate mode is obtained by rotation and superposition of fundamental mode. A triangular patch bandpass filter with multi-mode operation employing higher order modes that utilized fractal defection is realized [1112]. An isosceles triangular patch resonator (ITPR) is presented in [13] with corneres cut and slits to realize wideband bandpass filter operating in X-band. A rightangled triangular patch resonator with fractal hole is used to achieve single and dual-band bandpass responses [14]. Different types of DGS resonators are also used to realize selective and tunable bandpass filters as detailed in [15-18].

Very few asymmetric filters are designed due to difficulties in controlling and arranging cross couplings in planar configuration and still there is a scope to realize such high selectivity filters. In this paper, two-pole and four-pole filters are designed using microstrip triangular patch employing horizontal and vertical slots. These slots are used to excite dual degenerate modes and also to control the position of transmission zeros on either side of the pass band. These slots will change the direction of the current flowing on the surface of the patch resulting in transmission zero switching from lower passband to upper passband. The rest of the paper is organized as follows. Section II explains with design characteristics of dual-mode triangular patch and its surface current distribution. Prototype filters are designed and simulated, in section III, with first filter exhibiting transmission zero on lower side of the passband, second filter showing transmission zero on upper side of the passband and third filter displays transmission zeros on both sides of the passband. Experimental results are presented in section IV. Finally, conclusions are given in section V.

\section{Dual-mode Resonator}

According to Wheeler's cavity model, surrounded by magnetic walls, there are no electromagnetic field variations along the thickness of the substrate direction. Electric field $E_{Z}$ satisfies Helmholtz wave equation [19], 


$$
\begin{gathered}
\left(\frac{\partial}{\partial \mathrm{x}^{2}}+\frac{\partial}{\partial \mathrm{y}^{2}}+k_{m, n, l}^{2}\right) E_{Z}=0 \\
k_{m, n, l}=\frac{4 \pi}{3 a} \sqrt{m^{2}+m n+n^{2}}
\end{gathered}
$$

Where $E_{Z}$ is given as $A_{m, n, l} T(x, y), A_{m, n, l}$ is constant based on the magnetic boundary conditions, $k_{m, n, l}$ denotes wave-number constant and $T(x, y)$ is given as

$$
\begin{aligned}
& T(x, y)=\cos \left[\left(\frac{2 \pi x}{\sqrt{3} a}+\frac{2 \pi}{3}\right) l\right] \cdot \cos \left[\frac{2 \pi(m-n) y}{3 a}\right] \\
& +\cos \left[\left(\frac{2 \pi x}{\sqrt{3} a}+\frac{2 \pi}{3}\right) m\right] \cdot \cos \left[\frac{2 \pi(n-l) y}{3 a}\right]+ \\
& \cos \left[\left(\frac{2 \pi x}{\sqrt{3} a}+\frac{2 \pi}{3}\right) n\right] \cdot \cos \left[\frac{2 \pi(l-m) y}{3 a}\right] \\
& E_{z}=A_{m, n} T(x, y), H_{x}=\frac{j}{\omega \mu_{o} \mu_{r}} \frac{\partial E_{z}}{\partial y} \\
& H_{y}=\frac{-j}{\omega \mu_{o} \mu_{r}} \frac{\partial E_{z}}{\partial x}, E_{x}=E_{y}=H_{z}=0
\end{aligned}
$$

The resonant frequencies of dominant mode and higher order modes of the triangular patch are given by

$$
\begin{aligned}
& f_{1,0,-1}=\frac{2 c}{3 a \sqrt{\varepsilon_{r}}} \\
& f_{m, n, l}=\frac{2 c}{3 a \sqrt{\varepsilon_{r}}} \sqrt{m^{2}+m n+n^{2}}
\end{aligned}
$$

Where , $\mathcal{C}^{\prime}$ is the velocity of light,, $\mathcal{E}_{r}{ }^{c}$ is the relative dielectric constant of the substrate, and, $a^{\text {ee }}$ is the side dimension of the triangle. We have chosen , $a^{\text {ee }}$ as $12 \mathrm{~mm}$, which gives the fundamental resonant frequency of 5.16 $\mathrm{GHz}$ for dominant mode with $m=1, n=0$.

The equilateral triangular patch is known to exhibit dual-mode property [10]. In a triangular patch resonator the coupling of two degenerated modes can be done by disturbing the symmetry of the resonator or by adding slot in the structure. The dual degenerate mode is obtained by rotating the other mode to $90^{\circ}$ in the coordinate system and superposition of fundamental mode. Unlike the square and circular patches, these degenerate modes do not couple to each other directly, but couple to input and output ports by creating two parallel paths from input to output. The coupling arrangement is shown in Figure 1. These two modes are also referred to as even and odd modes. A fullwave HFSS Eigen mode simulator is used for simulating the structures. RT duriod substrate with a dielectric constant of 10.2 and with a height of $0.635 \mathrm{~mm}$ is used for simulating the structures. The surface current distribution for even and

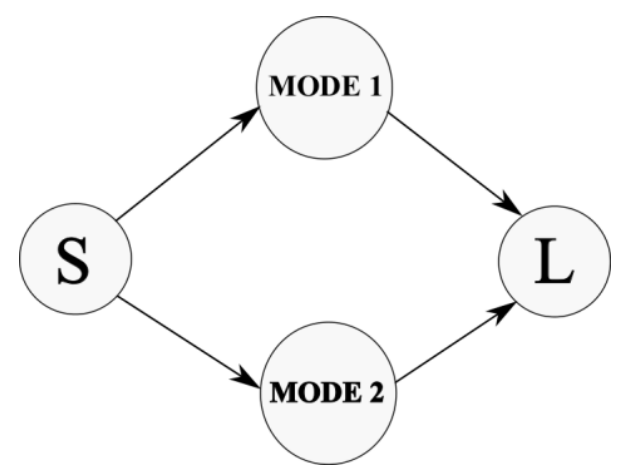

Figure 1: Coupling arrangement between dual degenerate modes of a triangular patch.

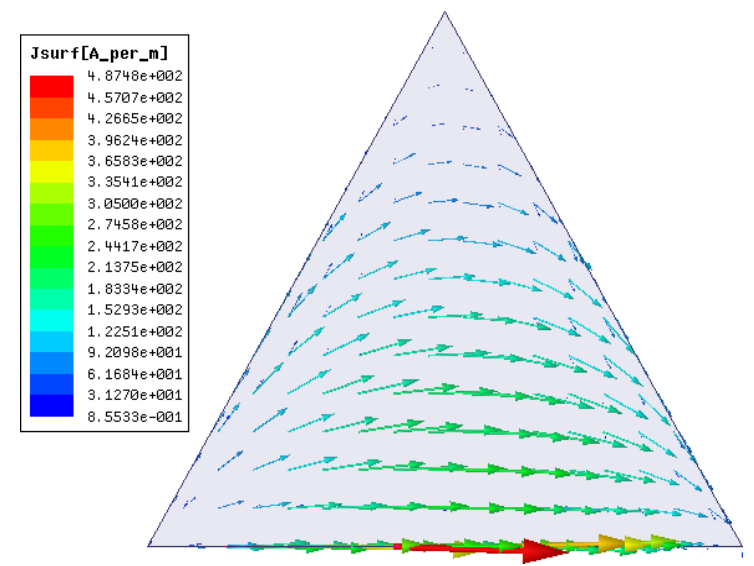

(a)

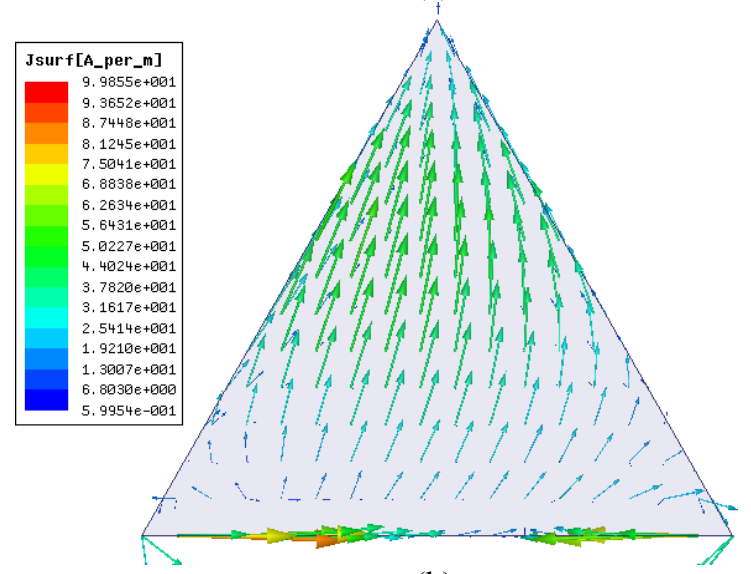

(b)

Figure 2: Surface current distribution of triangular patch (a) odd mode, (b) even mode.

an odd mode is depicted in Figure 2. For even mode, current is concentrated in the upper part of the patch along the slant sides of the triangle, whereas for odd mode, current is concentrated around base of the triangle. The surface current of the even mode can be reoriented by adding a horizontal slot near to the base of the patch resonator and similarly the odd mode current can be reoriented by adding a vertical slot near to the top of the patch resonator. The orientation of surface currents for specified horizontal and vertical slots is shown in Figure 3. From the Figure 3, it is observed that horizontal slot does not disturb odd mode, 
whereas it perturbs the field of even mode. Similarly a vertical slot does not disturb even mode, but it will alter the field of odd mode. We can notice that the direction of the current flowing on the surface of the patch is reversed, when we change from horizontal to vertical slot. This rerouting of surface current distribution leads to switching of transmission zeros.

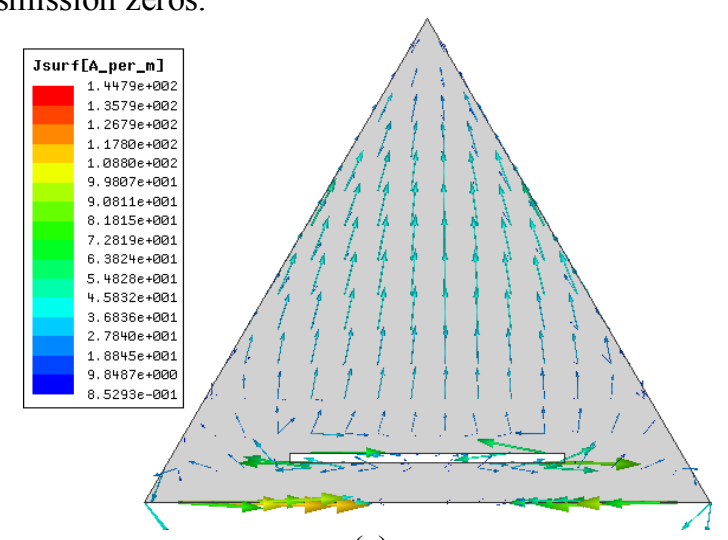

(a)

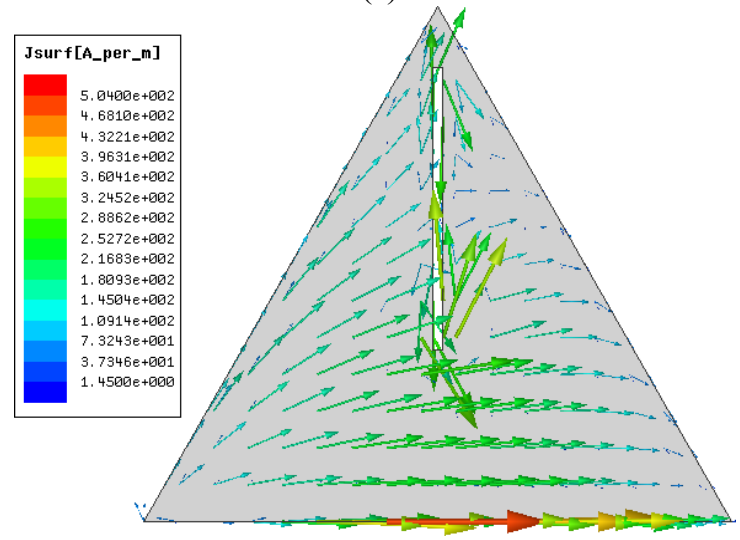

(b)

Figure 3: Surface current distribution of triangular patch after adding slots (a) horizontal slot (b) vertical slot.

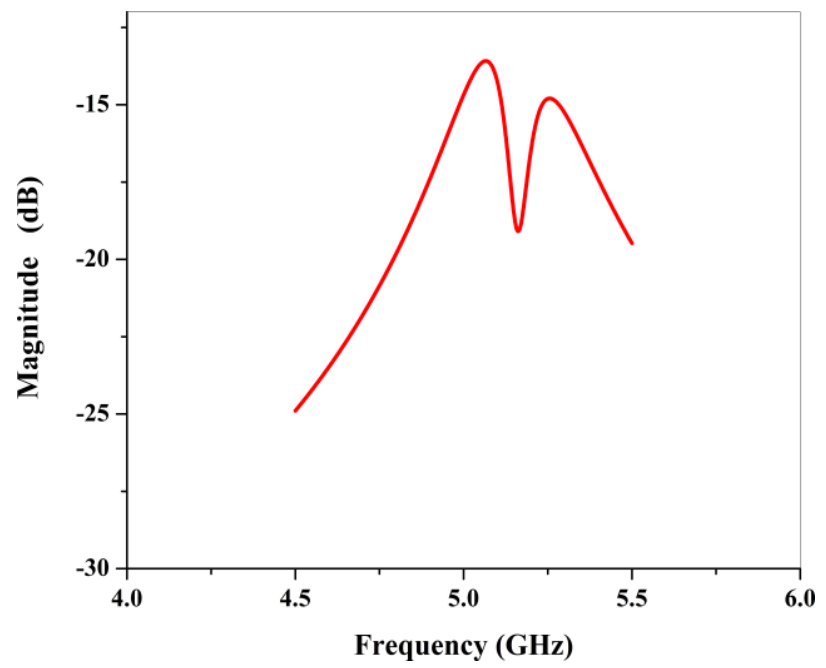

Figure 4: Splitting of dual modes of the patch resonator.

The splitting of dual modes can be done by deforming the equilateral triangle to isosceles or cutting the edges of the triangle. It is also achieved by incorporating slots over the surface of the patch. The dual resonant modes under weak coupling are shown in Figure 4. The resonant frequencies of degenerated modes can be controlled by varying the slot lengths. Figures 5(a) and 5(b) shows the variation of resonant frequencies of odd and even modes with respect to variation in the horizontal and vertical slot lengths. From Figure 5(a), it is observed that by increasing the length of the horizontal slot, the resonant frequencies of even mode are shifting from $5.05 \mathrm{GHz}$ to $5.25 \mathrm{GHz}$ while the odd mode is unchanged. From Figure 5(b), it is found that variation of vertical slot affecting the odd mode frequencies whereas even mode frequencies are hardly affected.

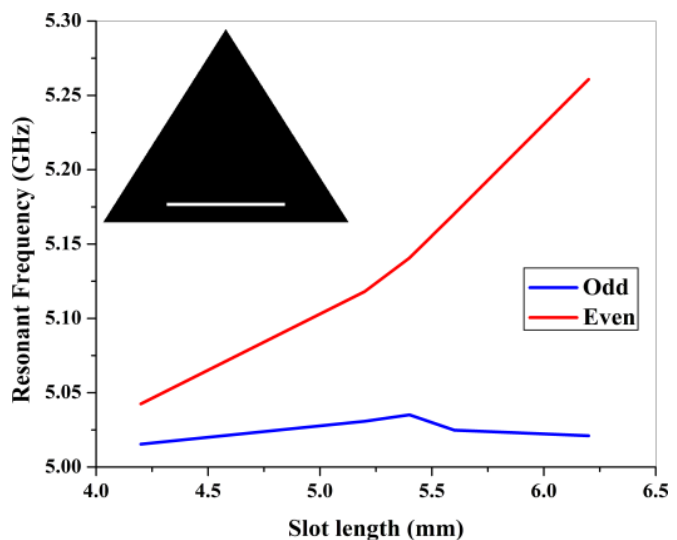

(a)

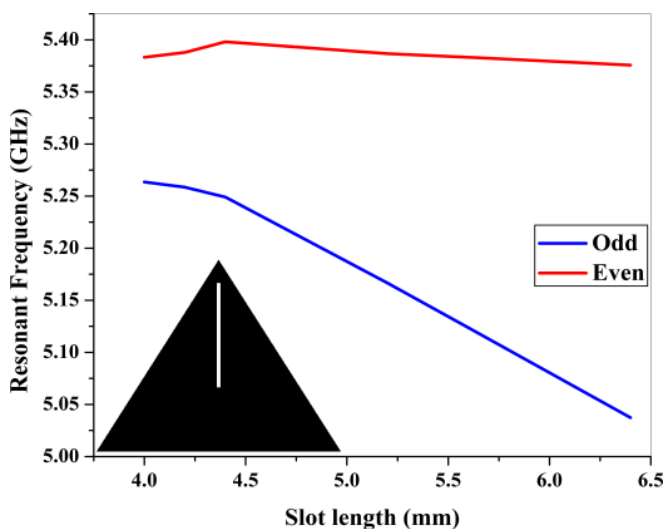

(b)

Figure 5: Separation of dual resonant frequencies with (a) horizontal slot and (b) vertical slot.

\section{Bandpass Filter Design}

Based on the above discussions and simulations, two dualmode and one quadruple-mode filters are designed. All the filters are fed by $50 \Omega$ feed lines at the bottom of the triangular patch with parallel coupling. Earlier reported work [9] uses cascading of triangular patch resonators with changing the position of feed lines to achieve selective filters. In contrast to slant line feed, the proposed design utilizes hemline feed, and also slots are used to position the transmission zeros on either side of the center frequency using a single equilateral triangular patch, without changing 
the position of feed lines. Figure 6(a) shows the first dualmode filter (Filter A), which uses horizontal slot placed at the bottom side of patch. Figure 6(b) depicts second dualmode filter (Filter B), which employs vertical slot placed at the top side of the patch. Figure 6(c) illustrates quadruplemode filter which utilizes both horizontal and vertical slots. The structural parameters of all the designed filters are given in Table 1. The side dimension of the triangular patch , a $\mathrm{a}^{\text {ee }}$ is chosen as $12 \mathrm{~mm}$. All the three filters are designed using commercially available full-wave EM simulator High Frequency Structure Simulator.

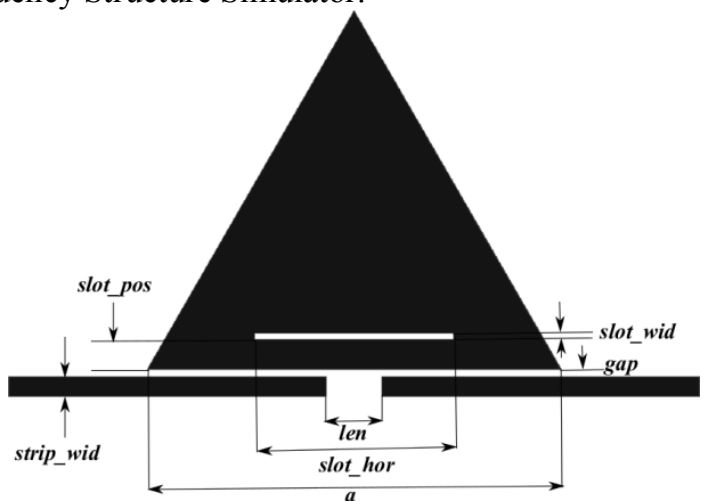

(a) Filter $\mathrm{A}$

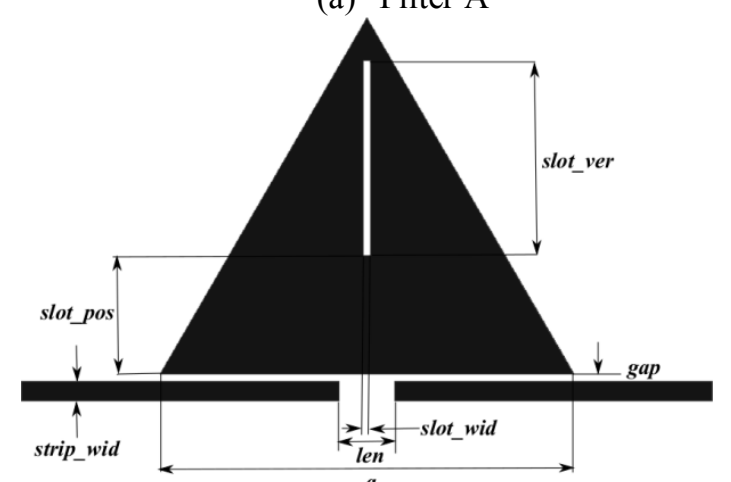

(b) ${ }^{a}$ Filter B

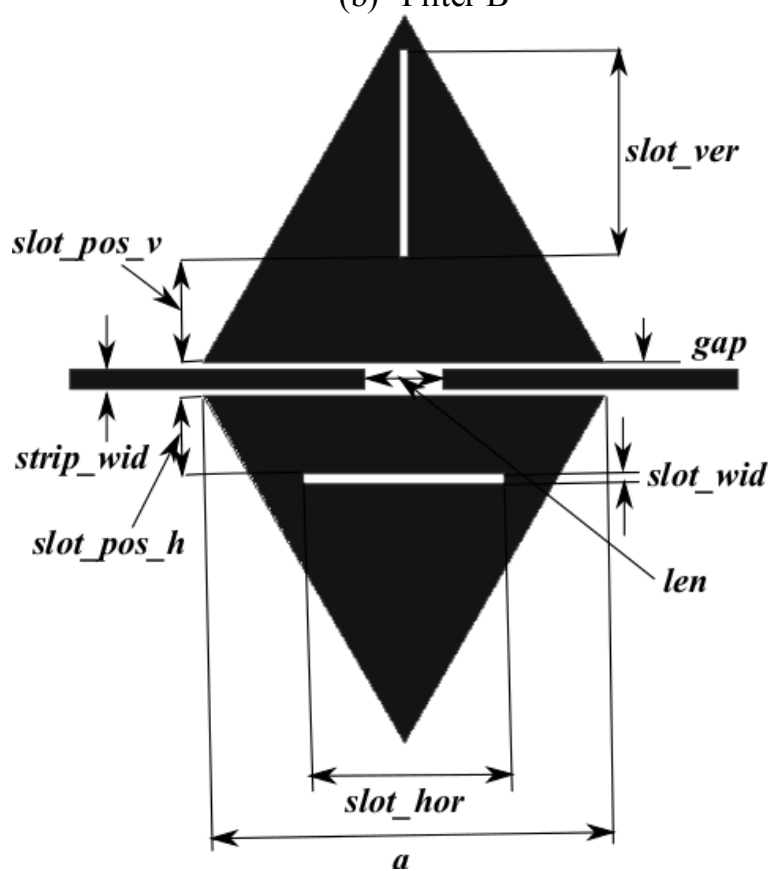

(c) Filter C
Figure 6: (a) Two-pole bandpass filter using horizontal slot (Filter A), (b) two-pole bandpass filter using vertical slot (Filter B), (c) four-pole filter using both horizontal and vertical slots (Filter C).

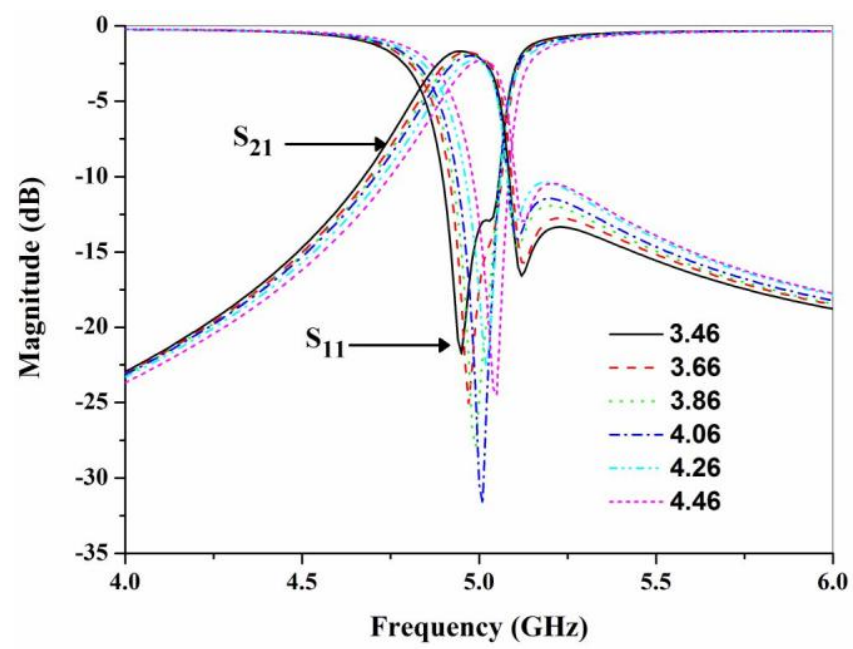

Figure 7: Simulation results for various positions (slot_pos) of horizontal slot for a slot length (slot_hor) of $5.8 \mathrm{~mm}$.

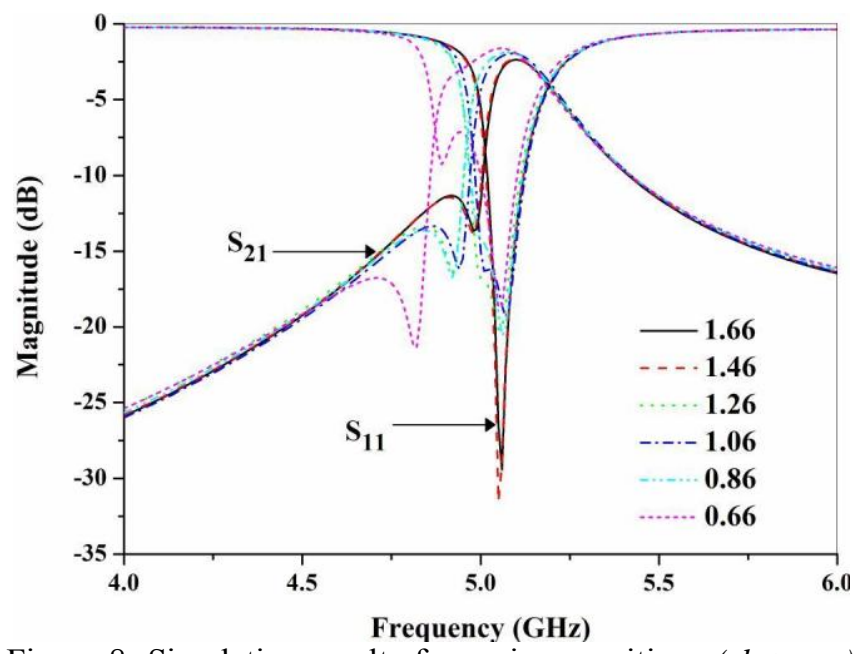

Figure 8: Simulation results for various positions (slot_pos) of vertical slot for a slot length (slot_ver) of $5.7 \mathrm{~mm}$.

The length and position of both horizontal and vertical slots is optimized by using full wave EM simulator. The lengths of slots are approximately around $\lambda / 10$. The position of slots affects both insertion and return loss response of the filter. Figure 7 shows the simulated results for various slot positions of horizontal slot, varied above with respect to the slot position shown in Figure 6(a), keeping length of the horizontal slot fixed at $5.8 \mathrm{~mm}$ in steps of $0.2 \mathrm{~mm}$. Similarly Figure 8 depicts the simulation results for various positions of vertical slot, varied above with respect to the slot position shown in Figure 6(b), keeping length of vertical slot constant by $5.7 \mathrm{~mm}$ in steps of 0.2 $\mathrm{mm}$. The slot positions are chosen in such way that both insertion and return loss behave well, and the corresponding slot positions are given in Table 1 . 
Table 1: Dimensions of the filter structures (mm).

\begin{tabular}{|c|c|c|c|c|c|c|c|c|c|}
\hline $\begin{array}{c}\text { Filter } \\
\text { type }\end{array}$ & strip_wid & slot_wid & slot_hor & slot_ver & Gap & len & slot_pos & slot_pos_h & slot_pos_v \\
\hline A & 0.59 & 0.2 & 5.8 & ---- & 0.2 & 1.2 & 0.86 & ---- & --- \\
\hline B & 0.59 & 0.2 & ---- & 5.7 & 0.2 & 0.8 & 3.46 & ---- & --- \\
\hline C & 0.59 & 0.2 & 6.0 & 6.0 & 0.2 & 1.2 & ---- & 2.31 & 3.17 \\
\hline
\end{tabular}

\section{Results}

The filters described above are fabricated using RT Duriod substrate with dielectric constant of 10.2 and substrate height of $0.635 \mathrm{~mm}$. Copper cladding is used with a thickness of $0.035 \mathrm{~mm}$. The photographs of the fabricated

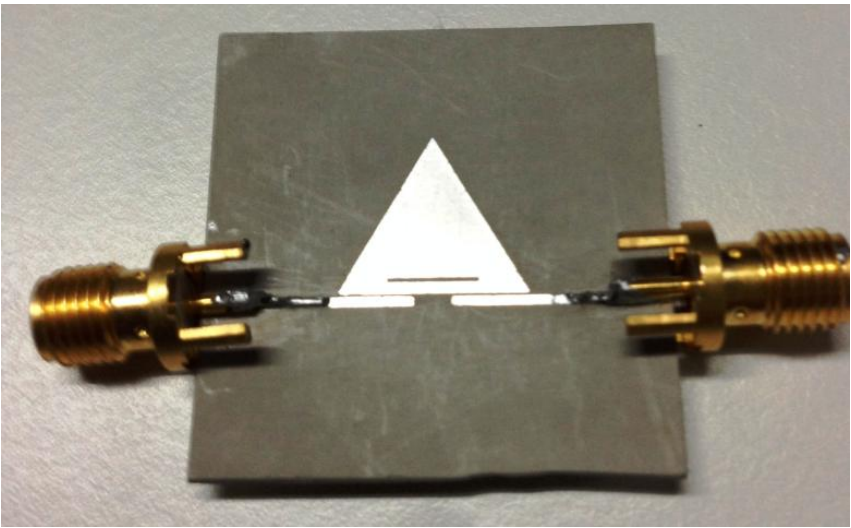

(a) Filter $\mathrm{A}$

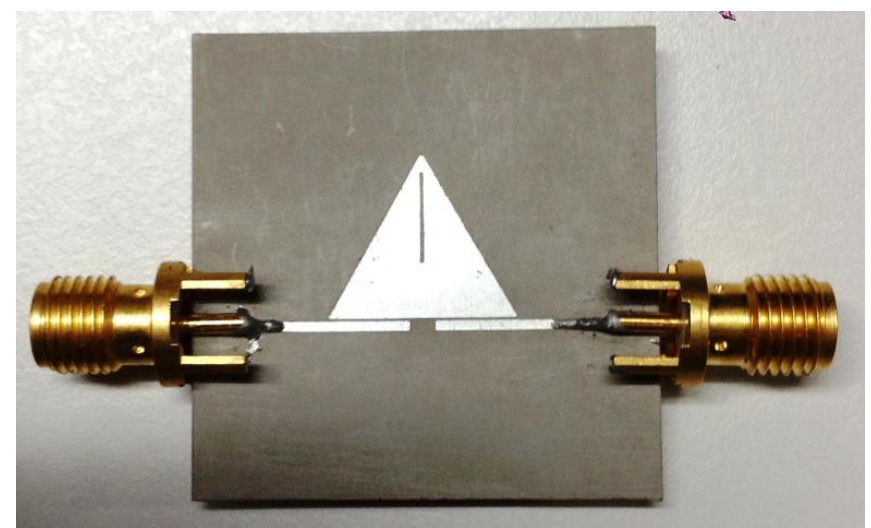

(b) Filter B

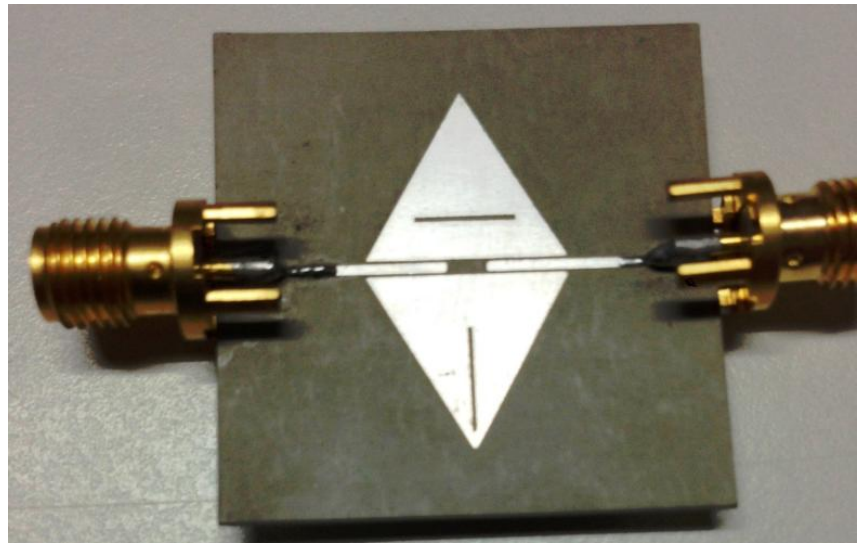

(c) Filter $\mathrm{C}$

Figure 9: Photographs of the fabricated two pole and four pole filters.

filters are shown in Figure 9. Agilent"s N99181A series vector network analyzer is used to measure the results. Figure 9(a) shows the dual-mode filter (Filter A) with horizontal slot and the filter is resonating at $5.06 \mathrm{GHz}$. The simulated and measured results of return loss and insertion loss are shown in Figure 10. From Figure 10(b) it is observed that there is a transmission zero at the lower passband of the filter which is at a frequency of $4.91 \mathrm{GHz}$. The simulation results indicate an insertion loss of $1.93 \mathrm{~dB}$ and return loss of $20.58 \mathrm{~dB}$ with a fractional bandwidth of $4.94 \%$. The measured insertion and return loss are at 2.27 $\mathrm{dB}$ and $20.58 \mathrm{~dB}$ respectively. 


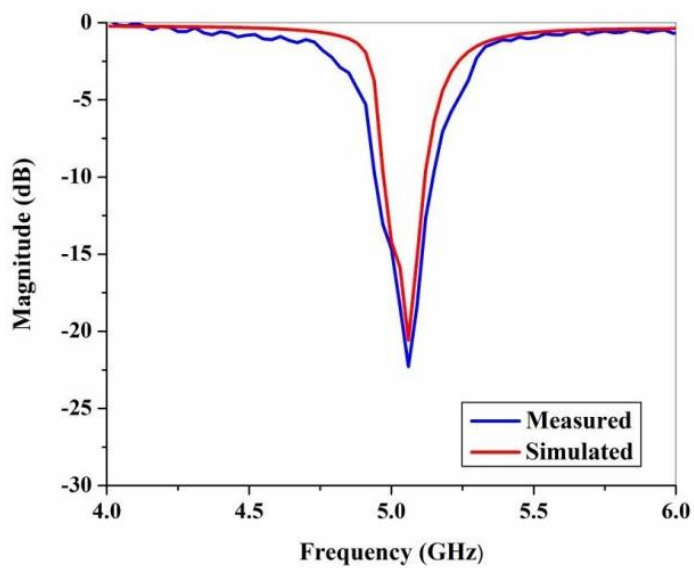

(a)

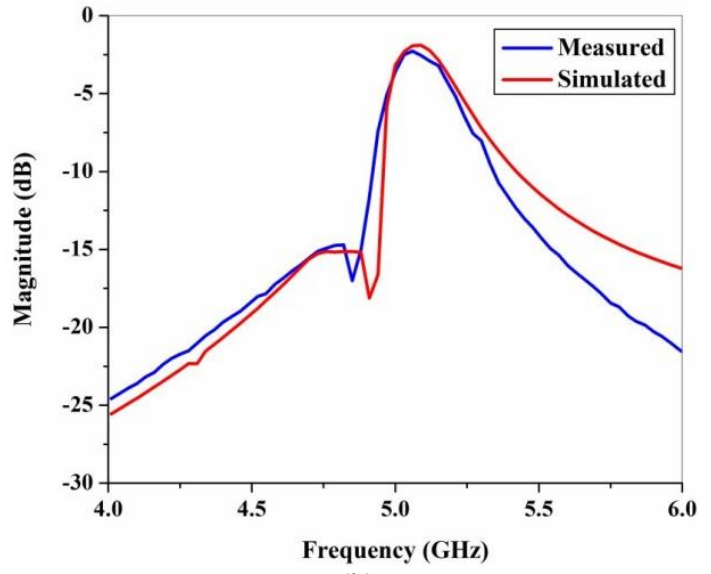

(b)

Figure 10: Simulated and measured results of bandpass filter using horizontal slot (Filter A) (a) return loss, (b) insertion loss.

A transmission zero at the upper passband can be introduced by replacing the horizontal slot with a vertical slot. Figure 9(b) shows the second dual-mode filter (Filter B) with a vertical slot and the filter produces transmission zero at the upper passband. Figure 11(a) and 11(b), shows the simulated and measured return loss and insertion loss response of the Filter B. Simulation results show that the center frequency of the filter $\mathrm{B}$ is at $4.95 \mathrm{GHz}$ with an insertion loss of $1.67 \mathrm{~dB}$ and return loss of $21.78 \mathrm{~dB}$. The fractional bandwidth of the filter is $4.84 \%$. The measured insertion and return loss are at $2.48 \mathrm{~dB}$ and $12.69 \mathrm{~dB}$ respectively. The generation of transmission zeros on either side of the center frequency i.e. upper or lower passband will produce very high selectivity on the respective passband which is a major requirement for many applications like diplexers and multiplexers. Bandpass filter with transmission zeros on both sides of the passband resembles the ideal filter response which results in high attenuation on both sides of the stopband. A four-pole filter with transmission zeros on both sides of the passband is shown in Figure 9(c). Here we have combined the two patch

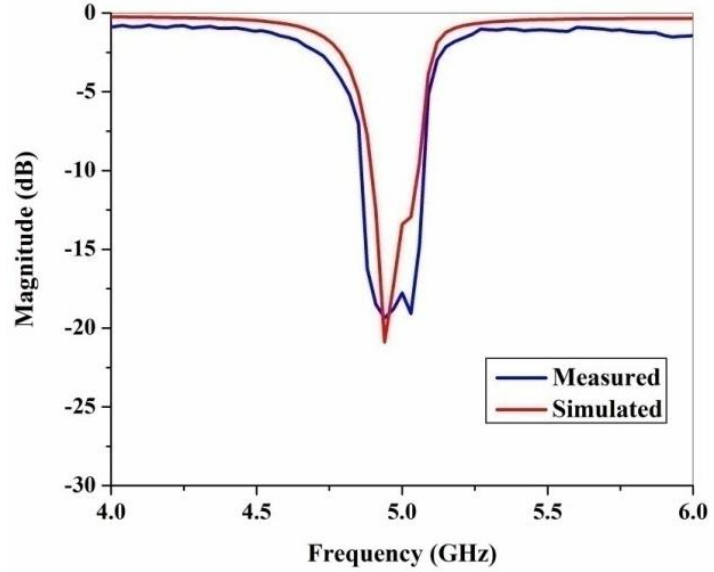

(a)

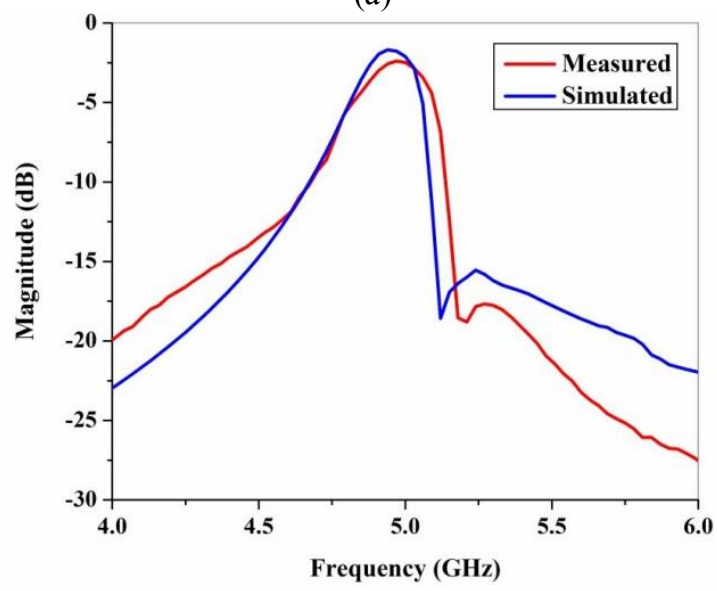

(b)

Figure 11: Simulated and measured results of bandpass filter using vertical slot (Filter B) (a) return loss, (b) insertion loss.

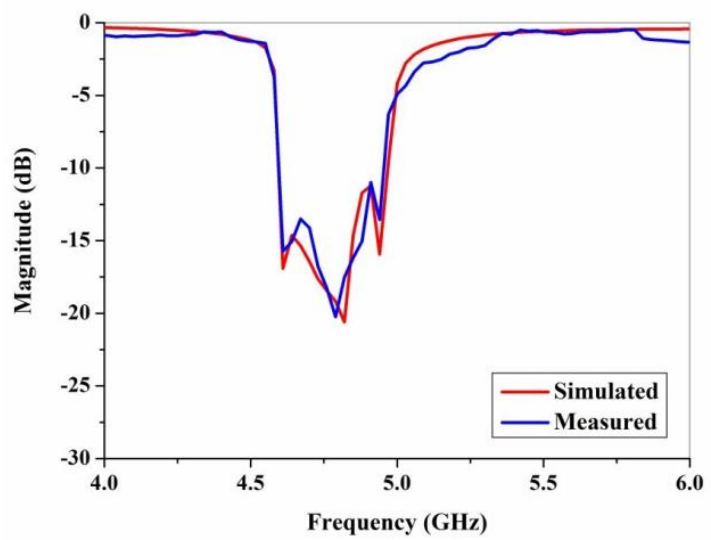

(a) 


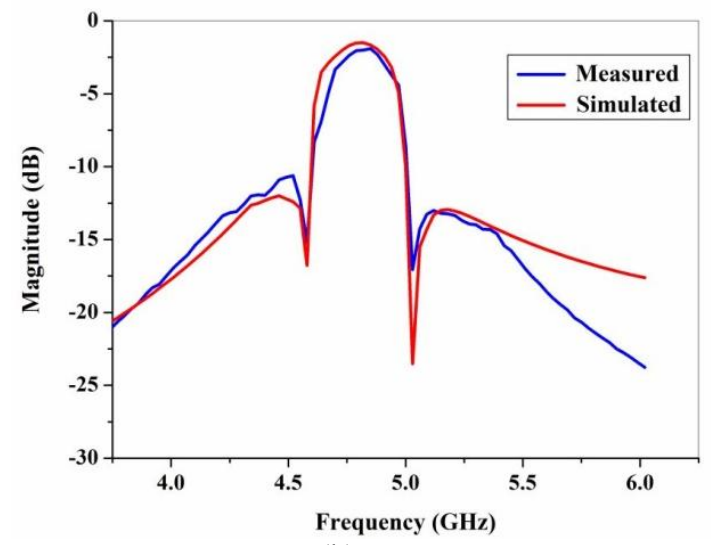

(b)

Figure 12: Simulated and measured results of bandpass filter using both horizontal and vertical slots (Filter C) (a) return loss, (b) insertion loss.

resonators with horizontal and vertical slots to obtain transmission zeros on both sides of passband. From the simulated the measured results shown in Figure 12, the filter

Table 2. Performance analysis of the designed filters with earlier reported patch resonator filters.

\begin{tabular}{|c|c|c|c|c|c|c|}
\hline $\begin{array}{l}\text { Reference } \\
\text { No. }\end{array}$ & Patch Topology & $\begin{array}{l}\text { Side } \\
\text { dimen } \\
\text { sions } \\
(\mathrm{mm})\end{array}$ & $\begin{array}{l}\text { Center } \\
\text { frequency } \\
(\mathrm{GHz})\end{array}$ & $\begin{array}{l}\text { Insertion } \\
\text { Loss } \\
(\mathrm{dB})\end{array}$ & $\begin{array}{c}\text { Fractional } \\
\text { Bandwidth } \\
\text { (FBW) }\end{array}$ & $\begin{array}{l}\text { Frequency } \\
\text { Response }\end{array}$ \\
\hline [20] & Square & 20 & 1.62 & 3.4 & 1.6 & Symmetric \\
\hline [9] & $\begin{array}{l}\text { Triangular [I] } \\
\text { Triangular [II] }\end{array}$ & 15 & 4.1 & ------ & ------ & Asymmetric \\
\hline [21] & $\begin{array}{c}\text { Star type [I] } \\
\text { Star type [II] }\end{array}$ & 15 & $\begin{array}{l}2.98 \\
2.98\end{array}$ & $\begin{array}{l}2 \\
2\end{array}$ & $\begin{array}{l}4.99 \\
4.69\end{array}$ & Asymmetric \\
\hline [22] & Circular & 36 & 1.58 & 1.41 & 20.25 & Symmetric \\
\hline [14] & Triangular & 5.7 & 10.36 & 2.08 & 55 & Symmetric \\
\hline This work & $\begin{array}{c}\text { Triangular [I] } \\
\text { Triangular [II] } \\
\text { Triangular [III] }\end{array}$ & 12 & $\begin{array}{l}5.06 \\
4.95 \\
4.82\end{array}$ & $\begin{array}{l}2.27 \\
2.48 \\
2.40\end{array}$ & $\begin{array}{l}4.94 \\
4.84 \\
6.43\end{array}$ & $\begin{array}{l}\text { Asymmetric \& } \\
\text { Symmetric }\end{array}$ \\
\hline
\end{tabular}

\section{Conclusion}

Three highly selective bandpass filters are designed using dual-mode triangular patch resonators. Horizontal and vertical slots are used to produce transmission zeros on either side of passband. Degenerated modes are coupled by varying the slot positions and dimensions. Two-pole filters have transmission zeros on one side of the passband whereas quadruple-mode filter is more selective with transmission zeros on both sides of the passband. From the measured results, it is observed that insertion loss and return loss response of all filters are close to bandwidth of less than $7 \%$ associated with high selectivity and high power handling capability. 


\section{Acknowledgement}

The authors are thankful to Bapuji Institute of Engineering \& Technology, Davangere, Karnataka, India, for their kind support and encouragement towards the successful completion of this work.

\section{References}

[1] R.R Mansour, "Design of superconductive multiplexers using single mode and dual mode filters," IEEE Trans. Microwave Theory Tech., Vol. 42, pp. 1411- 1418, 1994.

[2] J.S. Hong and M.J. Lancaster, "Microstrip cross-coupled trisection bandpass filters with asymmetric frequency characteristics," IEE Proc. Microwave, Antennas Propag., Vo. 146, pp. 84-90, 1999.

[3] G.M. Erylimaz, C. Karpuz and A. Gorur, "Dual-Mode microstrip filters with adjustable transmission zeros," IET Microw. Antennas Propag., Vol. 2, pp. 839-847, 2008.

[4] I. Wolff, "Microstrip bandpass filter using degenerate modes of a microstrip ring resonator," Electronic Lett., Vol. 8, pp. 302-303, 1972.

[5] J.S. Hong and M.J. Lancaster, "Bandpass characteristics of new dual mode microstrip square loop resonators," Electronics Lett., Vol. 31, pp. 891-892, 1995.

[6] C. Lugo and J. Papapolymerou, "Bandpass filter design using a microstrip triangular loop resonator with dual mode operation," IEEE Microwave Wireless Compo. Lett., Vol. 15, pp. 475-477, 2005.

[7] J.S. Hong and M.J. Lancaster, "Microstrip bandpass filter using degenerate modes of a novel meander loop resonator," IEEE Microwave Guided wave Lett., Vol. 2, pp. 371-372, 1995.

[8] J.A. Curtis and S.J. Fiedziuszko, "Miniature dual mode microstrip filters," IEEE MTT-S Dig., pp. 443-446, 1991.

[9] J.S. Hong and M.J. Lancaster, "Microstrip triangular patch resonator filters," IEEE MTT-S Dig., pp. 331-334, 2001.

[10] J.S. Hong and Shuzhou Li, "Theory and experiment of dual mode microstrip triangular patch resonators and filters," IEEE Trans. Microwave Theory Tech., Vol. 52, pp. 1237-1243, 2004.

[11] J.-K. Xiao, Q.-X. Chu and S. Zhang, "Novel microstrip triangular resonator bandpass filter with transmission zeros and wide bands using fractal-shaped defection," Progress in Electromagnetic Research, Vol. 77, pp. 343-356, 2007.
[13] J.-K. Xiao, Q.-X. Chu and H.-F. Huang, "Triangular resonator bandpass filter with tunable operation," Progress in Electromagnetic Research, Vol. 2, pp. 167-176, 2008.

[14] A.R.H. Alahwari.and A. Ismail, "Compact wideband bandpass filter using single corners-cut isosceles triangular patch resonator," Progress in Electromagnetic Research C, Vol. 14, pp. 227-237, 2010.

[15] A. Boutejdar, A. Elsherbini, A. Balalem, J. Machacand A. S. Omar, "Design of new DGS hairpin microstrip bandpass filter using coupling matrix method", Proc. PIERS'2007, Prague, pp. 261-265, 2007.

[16] A. Boutejdar, A.A. Ibrahim, W.A.E. Ali, "Design of compact size and tunable bandpass filter for WLAN applications," Electronics Letters, Vol. 52, No. 24, pp. 19961997, 2016

[17] A. Boutejdar, "A new approach to design compact tunable BPF starting from simple LPF topology using a single T-DGS-resonator and ceramic capacitors", Microwave and Optical Technology Letters, Vol. 58, No.5, pp. 1142-1148, 2016.

[18] A. Boutejdar, "Design of 5 GHz-compact reconfigurable DGS-bandpass filter using varactor-diode device and coupling matrix technique, Microwave and Optical Technology Letters, Vol, 58, No. 2, pp. 304-309, 2016.

[19] R. Garg, P. Bhartia, I. Bahl, Microstrip Antenna Design Handbook, Boston-London: Artech House, 2001. pp. 429430.

[20] Lei Zhu, P-M Wecowski and $\mathrm{Ke} \mathrm{Wu}$, "New planar dual-mode filter using cross-slotted patch resonator for simultaneous size and loss reduction", IEEE Trans. Microw. Theory Tech., Vol. 47, No. 5, pp. 650-654, May 1999.

[21] S.R. Zinka, Akhilesh Mohan and Animesh Biswas, "Bandpass filter realization using degenerate dual-modes of a new type of patch resonator for significant size reduction", Proceedings of Asia Pacific Microwave Conference, pp. 1-4, 2007.

[22] Y.K. Singh and Ajay Chakrabarty, "Miniaturized dualmode circular patch bandpass filters with wide harmonic separation", IEEE Microw. Wireless Compo., Vol. 18, pp. 584-586, Sept. 2008. 